A

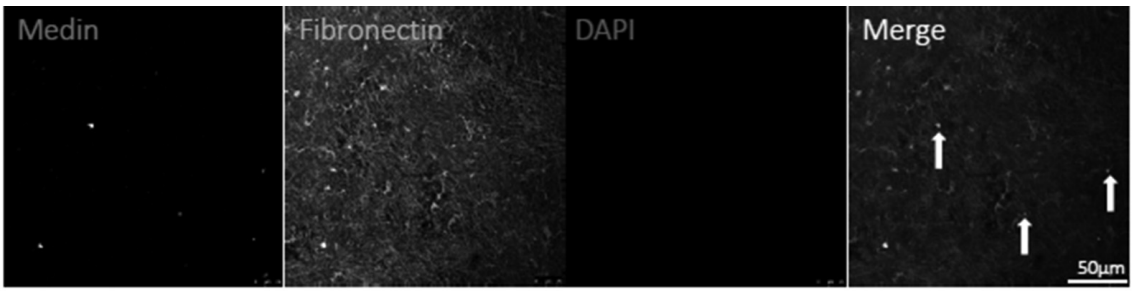

B

Control

$+\mathrm{CaP}$

$+\mathrm{CaP}+\mathrm{SOM}$
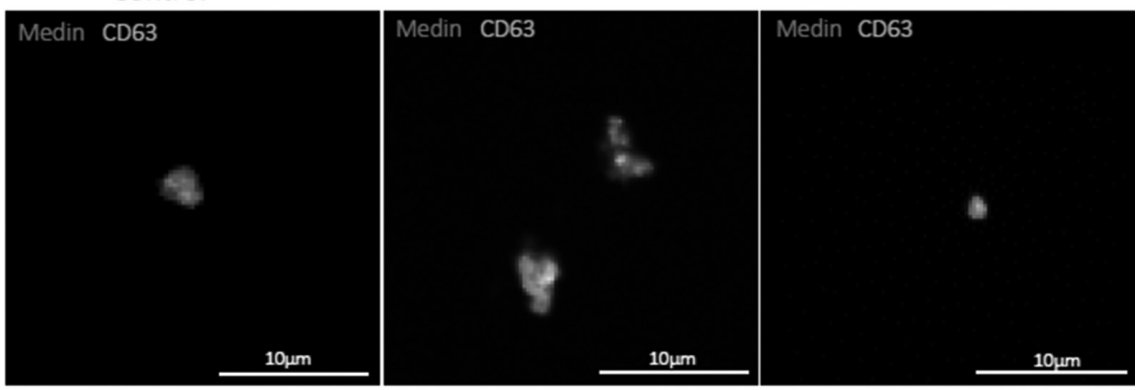

C

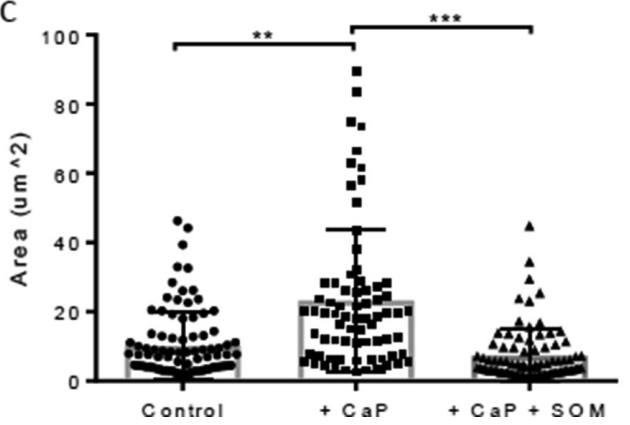

Abstract BS47 Figure 2 Medin in deposited in the ECM by exosomes. A) Deposits of medin are observed in ECM from cultured VMSCs (white arrows). Fibronectic staining shows the presence of ECM. B) Medin deposits colocalise with exosome marker, CD63. Treatment with calcium and phosphate $(+\mathrm{CaP})$ increases the size of medin deposits. Treatment with an exosome secretion inhibitor, spiroepoxide (+SOM), decreases the deposit size. D) The area of each medin aggregate was measured using ImageJ

and phosphate conditions. FACS was used for quantification of exosome secretion. Exosomes were isolated by differential ultracentrifugation. Extracellular matrix (ECM) was synthesised in vitro for immunofluorescence and Western blotting. Cresolphthalein assays were used to quantify calcification.

Results MFGE8, medin and calcification, shown by von Kossa staining, co-localised in the medial layer of aortas from old subjects (figure 1). Medin and calcium deposits appear predominantly extracellular. Exosome secretion is increased from senescent VSMCs and in calcifying conditions. MFGE8 was expressed by VSMCs and was secreted exclusively in exosomes and its expression and secretion increased in calcifying conditions. Immunofluorescence staining of the ECM in vitro showed medin deposits co-localise with CD63, an exosome marker. The size of medin deposits in the ECM was increased when cells were treated with calcium and phosphate and this effect was reduced with an exosome secretion inhibitor, spiroepoxide (figure 2). Treatment with spiroepoxide also decreased hydroxyapatite deposition in the ECM.

Conclusion Medin and MFGE8 are abundant in aged aortas and secreted by exosomes. The von Kossa and medin staining observed in the medial layer of aged subjects indicates colocalisation of AMA and calcification. Exosome secretion is increased with age as well as in calcifying conditions, which could contribute to the age-related deposition of medin in the
ECM. Blocking exosome release attenuates the effect of elevated calcium and phosphate on both the size of medin aggregates and the formation of hydroxyapatite in the ECM. This suggests VSMC-derived exosomes mediate both vascular calcification and medin aggregation; and conditions which induce vascular calcification also stimulate AMA formation.

Conflict of interest No conflict of interest

\section{BS48 MICRORNA EDITING IS INTEGRAL FOR INTERLEUKIN-6 TRANS-SIGNALLING AND LEUKOCYTE TRAFFICKING TO ISCHEMIC TISSUES}

${ }^{1}$ Aikaterini Gatsiou*, ${ }^{1}$ Simon Tual-Chalot, ${ }^{2}$ Francesca Bonini, ${ }^{3}$ Valeriana Cesarini, ${ }^{4}$ Almudena Ortega-Gomez, ${ }^{5}$ Kerida Shook, ${ }^{2}$ Jedrzej Hoffmann, ${ }^{6}$ Shin Kwak, ${ }^{7}$ Craig H. Selzman, ${ }^{8}$ Maurizio Martini, ${ }^{2}$ Stefanie Dimmeler, ${ }^{3}$ Angela Gallo, ${ }^{7}$ Stavros Drakos, ${ }^{4}$ Oliver Soehnlein, ${ }^{9}$ Konstastinos Stellos. ${ }^{1}$ Cardiovascular Research Centre, Institute of Genetic Medicine, Newcastle University, Newcastle upon; ${ }^{2} J W$ Goethe University Frankfurt, Frankfurt, Germany; ${ }^{3}$ Ospedale Pediatrico Bambino Gesù IRCCS, Rome, Italy; ${ }^{4}$ LMU Munich, Munich, Germany; ${ }^{5}$ Cardiovascular Research Centre, Institute of Genetic Medicine, Newcastle University; ${ }^{6}$ University of Tokyo, Tokyo, Japan; ${ }^{7}$ University of Utah, Salt Lake City, USA; ${ }^{8}$ Università Cattolica del Sacro Cuore, Rome, Italy; ${ }^{9} 1$ Cardiovascular Research Centre, Institute of Genetic Medicine, Newcastle University

\subsection{6/heartjnl-2019-BCS.209}

Background /Aim: Adenosine to inosine RNA editing is an essential posttranscriptional RNA modification catalysed by 